ARTICLE

DOI: $10.1038 / s 41467-017-02441-z$

\title{
Extreme sensitivity to ultraviolet light in the fungal pathogen causing white-nose syndrome of bats
}

Jonathan M. Palmer (10 ${ }^{1}$, Kevin P. Drees ${ }^{2}$, Jeffrey T. Foster (D) ${ }^{2,3}$ \& Daniel L. Lindner (iD ${ }^{1}$

Bat white-nose syndrome (WNS), caused by the fungal pathogen Pseudogymnoascus destructans, has decimated North American hibernating bats since its emergence in 2006. Here, we utilize comparative genomics to examine the evolutionary history of this pathogen in comparison to six closely related nonpathogenic species. P. destructans displays a large reduction in carbohydrate-utilizing enzymes (CAZymes) and in the predicted secretome $(\sim 50 \%)$, and an increase in lineage-specific genes. The pathogen has lost a key enzyme, UVE1, in the alternate excision repair (AER) pathway, which is known to contribute to repair of DNA lesions induced by ultraviolet (UV) light. Consistent with a nonfunctional AER pathway, $P$. destructans is extremely sensitive to UV light, as well as the DNA alkylating agent methyl methanesulfonate (MMS). The differential susceptibility of $P$. destructans to UV light in comparison to other hibernacula-inhabiting fungi represents a potential "Achilles' heel" of P. destructans that might be exploited for treatment of bats with WNS.

\footnotetext{
${ }^{1}$ Center for Forest Mycology Research, Northern Research Station, US Forest Service, Madison, WI 53726, USA. ${ }^{2}$ Department of Molecular, Cellular, and Biomedical Sciences, University of New Hampshire, Durham, NH 03824, USA. ${ }^{3}$ Present address: Pathogen and Microbiome Institute, Northern Arizona University, Flagstaff, AZ, USA. Correspondence and requests for materials should be addressed to D.L.L. (email: dlindner@fs.fed.us)
} 
W hite-nose syndrome of bats (WNS) continues to decimate hibernating bat populations in North America. The fungal disease was first documented in 2006 in eastern North America (New York) and the fungus has advanced west across the continent, most recently being detected on the West coast of North America ${ }^{1}$. WNS can result in $>90 \%$ mortality in local hibernating bat populations ${ }^{2}$ and is caused by the psychrophilic fungus Pseudogymnoascus destructans ${ }^{3,4}$. The fungus has a strict temperature growth range of $\sim 4-20^{\circ} \mathrm{C}$ and therefore can only infect bats during hibernation ${ }^{5}$. WNS is not a systemic infection, but rather is characterized by $P$. destructans colonization of the skin of hibernating bats, which manifests as cupping skin erosions based on histopathology ${ }^{6}$. Frequent arousal from hibernation, depletion of fat reserves, and dehydration appear to contribute to mortality in infected individuals ${ }^{7-10}$. $P$. destructans has been found throughout Eurasia and occasionally causes mild WNS symptoms; however, no mass mortality events have been observed in Eurasia ${ }^{11}$. P. destructans has spread in a "bulls eye" pattern in North America and has only been found in environments where WNS-infected bats are found, strongly suggesting that the fungus is not native to North America and represents a classic example of an introduced pathogen decimating a naïve population ${ }^{12,13}$. P. destructans is a member of the under-studied Pseudeurotiaceae family; while recent studies have resulted in several draft genome assemblies within this group, much of the biology of the fungal pathogen and its relatives remains unknown.

Here, we present functionally annotated genomes for $P$. destructans, as well as six closely related nonpathogenic Pseudogymnoascus species. Comparison of the pathogen with the nonpathogenic species provides an opportunity to obtain insight into the origins and adaptations of the fungal pathogen of WNS.

\section{Results}

Sequencing, assembly, and annotation. Recent phylogenetic work that aimed at identification and resolution of closely related species to $P$. destructans resulted in moving this species from genus Geomyces to Pseudogymnoascus ${ }^{4}$. Moreover, sampling for fungal isolates from hibernacular soil resulted in identification of other closely related Pseudogymnoascus species that are not known to be pathogenic ${ }^{12}$. We previously described the hybrid genome assembly of $P$. destructans ${ }^{14}$; in addition, we chose six closely related species and sequenced these isolates using Illumina chemistry, resulting in $\sim \times 250$ coverage for each genome (strains listed in Supplementary Table 1). All seven Pseudogymnoascus genomes were within the expected genome size for haploid ascomycetes ( 30-36 MB) with $\sim 50 \%$ GC content (Table 1). Genome annotation was completed using funannotate v0.1.8 (https://github.com/nextgenusfs/funannotate), resulting in 9335 protein-coding genome models for $P$. destructans and a range of 10,252-11,033 protein gene models for the nonpathogenic Pseudogymnoascus species (Table 1). Genome functional annotation (see "Methods" section) was added to each gene model; complete functional annotation is available in Supplementary Data 1-7.

Orthology and evolutionary phylogeny. Due to the importance of WNS, as well as interest in cold-tolerant fungi, there has recently been several draft genomes sequenced in the genus Pseudogymnoascus. To delineate relationships between these genomes, we acquired all Pseudogymnoascus genomes deposited in NCBI, as well as several outgroup species: Aspergillus nidulans, Botrytis cinerea, Fusarium fujikuroi, Penicillium chrysogenum, and Neurospora crassa (Supplementary Table 2). Single-copy BUSCO orthologs ${ }^{15}$ were extracted using Phyloma (https:// github.com/nextgenusfs/phyloma) and were used to generate a maximum likelihood phylogeny in RAxML v8.29 (PROTGAMMALG; 1000 bootstrap replicates) from 822 concatenated gene models. To estimate time of evolutionary divergence, node calibrations from Beimforde et al. ${ }^{16}$ were used in $\mathrm{r} 8 \mathrm{~s} \mathrm{v} 1.80^{17}$; Leotiomycetes-Sordariomycetes 267-430 MYA, Eurotiomycetes 273-537 MYA, Sordariomycetes 207-339 MYA, and Pezizomycotina 400-583 MYA (Supplementary Data 8). This analysis illustrates that the nonpathogenic Pseudogymnoascus species sequenced here are among the closest known relatives of $P$. destructans (Fig. 1). Additionally, these data suggested that the last known common ancestor of $P$. destructans diverged approximately 23.5 MYA. The oldest known chiropteran species in the fossil record, Palaeochiropteryx, was estimated to have lived 50-40 MYA $^{18,19}$, while the most recent adaptive speciation of Eurasian Myotis bat species occurred 9-6 MYA and the North American Myotis species emerged more recently at 6-3.2 $\mathrm{MYA}^{20}$. Thus, Eurasian Myotis species were present at the time when $P$. destructans diverged from its relatives, suggesting that it could have coevolved alongside modern-day Eurasian chiropteran species.

ProteinOrtho $5^{21}$ was used to identify 3949 single-copy orthologous groups between seven Pseudogymnoascus species used in this study, representing only $42.3 \%$ of the protein-coding genes in $P$. destructans. Relationships among these species were inferred from a maximum likelihood RAxML phylogeny based on the concatenated alignment of 500 orthologous proteins using Botrytis cinerea as an outgroup (Fig. 2). The orthologous proteome analysis identified 1934 unique proteins in $P$. destructans that were not found in any of the nonpathogenic Pseudogymnoascus species (Fig. 2 and Table 1), making $P$. destructans the species with the most unique proteins in our study. To identify orthologous proteins under positive selection, $\mathrm{d} N / \mathrm{d} S$ ratios for all orthologous groups were calculated using the codeml M0 model from PAML ${ }^{22}$. Likelihood ratio tests comparing the M1/M2 models, as well as M7/M8 models with significance at $<0.05$ were calculated to validate the estimated $\mathrm{dN} / \mathrm{d} S$ ratios greater than 1 , ratios that suggest positive selection is occurring 22 . Forty-six orthologous groups displayed evidence of positive selection. Of those orthologous groups under positive

Table 1 Genome summary statistics of $\boldsymbol{P}$. destructans and nonpathogenic Pseudogymnoascus species

\begin{tabular}{|c|c|c|c|c|c|c|c|c|c|c|c|c|}
\hline Species & Isolate & $\begin{array}{l}\text { Assembly } \\
\text { size }\end{array}$ & $\begin{array}{l}\text { Largest } \\
\text { scaffold }\end{array}$ & $\begin{array}{l}\text { Average } \\
\text { scaffold }\end{array}$ & $\begin{array}{l}\text { Num } \\
\text { scaffolds }\end{array}$ & $\begin{array}{l}\text { Scaffold } \\
\text { N50 }\end{array}$ & $\begin{array}{l}\text { Percent } \\
\text { GC }\end{array}$ & $\begin{array}{l}\text { Repetitive } \\
\text { DNA }\end{array}$ & $\begin{array}{l}\text { Num } \\
\text { genes }\end{array}$ & $\begin{array}{l}\text { Num } \\
\text { proteins }\end{array}$ & $\begin{array}{l}\text { Num } \\
\text { tRNA }\end{array}$ & $\begin{array}{l}\text { Unique } \\
\text { proteins }\end{array}$ \\
\hline P. destructans & $20631-21$ & $35,818,201$ & $2,552,699$ & 431,545 & 83 & $1,168,637$ & $49.24 \%$ & $38.17 \%$ & 9575 & 9335 & 240 & 1934 \\
\hline P. sp. 23342-1-11 & $23342-1-11$ & $32,900,320$ & 990,412 & 33,640 & 978 & 289,626 & $49.94 \%$ & $7.35 \%$ & 10,914 & 10,762 & 152 & 1275 \\
\hline P. sp. 24MN13 & 24MN13 & $30,179,533$ & 129,019 & 10,675 & 2827 & 24,078 & $50.17 \%$ & $3.45 \%$ & 10,368 & 10,269 & 99 & 1090 \\
\hline P. sp. WSF3629 & WSF3629 & $35,517,105$ & 741,106 & 86,839 & 409 & 189,864 & $48.85 \%$ & $10.78 \%$ & 11,193 & 11,033 & 160 & 1053 \\
\hline P. sp. 3VT5 & 03VT05 & $33,292,640$ & 361,480 & 38,893 & 856 & 115,508 & $49.09 \%$ & $15.94 \%$ & 10,393 & 10,252 & 141 & 795 \\
\hline P. sp. 5NY8 & 05NY08 & $32,206,663$ & 867,888 & 56,403 & 571 & 205,172 & $49.74 \%$ & $10.22 \%$ & 10,669 & 10,514 & 155 & 480 \\
\hline P. verrucosus & UAMH10579 & $30,174,856$ & $1,768,408$ & 199,833 & 151 & 446,342 & $50.36 \%$ & $4.60 \%$ & 10,715 & 10,573 & 142 & 515 \\
\hline
\end{tabular}




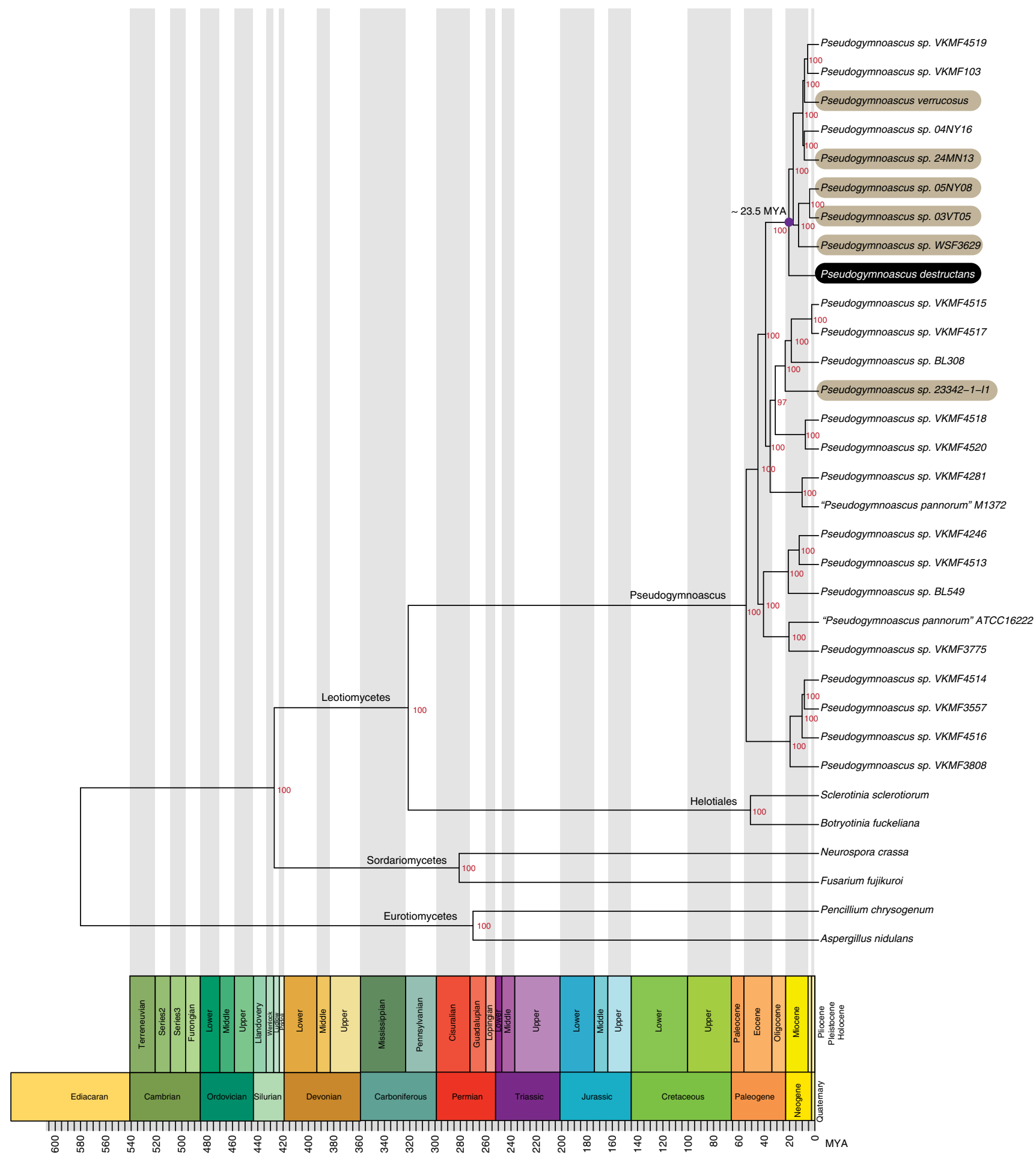

Fig. 1 Maximum likelihood phylogeny of Pseudogymnoascus draft genomes and several outgroup Ascomycetes calibrated using fossil evidence. The six nonpathogenic Pseudogymnoascus species sequenced here are among the closest known relatives of $P$. destructans. $P$. destructans was estimated to have diverged from its last common ancestor around 23.5 MYA. Node support values are derived from 1000 bootstrap replicates

selection, only 14 contained a protein from $P$. destructans (Supplementary Data 9). However, functional annotation for these orthologous groups did not provide sufficient information to predict a function for any of these orthologs.

Genome-level comparisons. Carbohydrate-activating enzymes (CAZymes) are a group of proteins involved in the breakdown and/or utilization of carbon. Genomes of fungi associated with plants, either plant pathogens or decomposers, generally harbor a greater number of CAZymes than those causing disease in ani$\mathrm{mals}^{23}$. The six nonpathogenic Pseudogymnoascus species on average harbored 493 CAZymes, ranging from 463 to 544; however, the genome of $P$. destructans contains only $~ 36 \%$ of the average number of CAZymes (179 in total) for the group (Fig. 2; Supplementary Data 10). The CAZymes can be further broken 


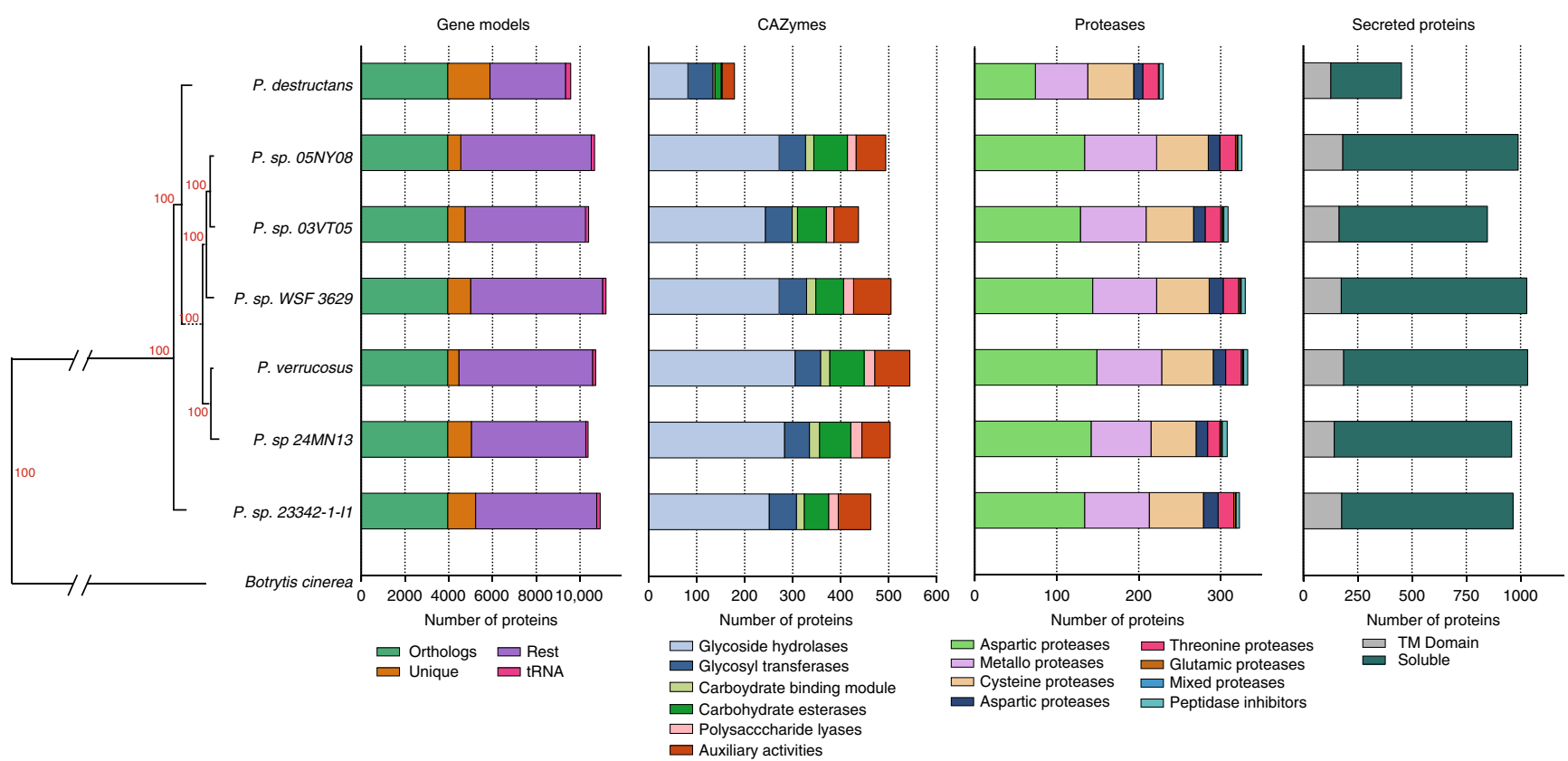

Fig. 2 Comparative genomic analyses of orthologous proteins of Pseudogymnoascus destructans and six close relatives. Maximum likelihood phylogeny illustrates relationships between fungi used in this study. Genome-level comparisons made based on number of gene models, number of CAZymes, number of proteases, and the number of secreted proteins as described in Methods

down into enzyme classes/modules. In comparison to the average of the nonpathogenic Pseudogymnoascus species, the genome of $P$. destructans harbors $\sim 69 \%$ (22) of auxiliary activity (AA) enzymes, $\sim 17 \%$ (4) of proteins containing carbohydrate-binding modules (CBM), $\sim 20 \%$ (13) of the carbohydrate esterases (CE), $\sim 30 \%$ (82) of the glycoside hydrolases $(\mathrm{GH}), \sim 10 \%$ (2) of the pectin lyases (PL), and $\sim 95 \%$ (52) of the glycosyltransferases (GT). Moreover, there were no specific CAZy families that were expanded in $P$. destructans that would indicate enhanced ability to utilize certain carbohydrate sources. Due to the large decrease in CAZymes, we hypothesized that $P$. destructans would have limited growth on complex and/or different carbon sources in comparison to the nonpathogenic Pseudogymnoascus species. To test this hypothesis, we compared growth on 190 carbon sources using a modified Biolog Phenotype Array Platform. Consistent with the large reduction in CAZymes in its genome, $P$. destructans was unable to utilize many carbon sources that the nonpathogenic Pseudogymnoascus species could readily utilize (Fig. 3, Supplementary Data 11). These data are consistent with a recent report comparing growth characteristics of $P$. destructans to closely related soil fungi ${ }^{24}$. The reduced CAZyme repertoire is a shared characteristic of other fungal pathogens of animals, including the dermatophytes, the true fungal dimorphic pathogens, as well as skin-inhabiting yeasts ${ }^{23}$. Presumably, the reduction in CAZymes reflects a change in carbon availability/ acquisition during the evolution from an ancestral saprobe to a bat pathogen, i.e., $P$. destructans may have shed CAZyme pathways necessary for living in soil/sediments but are not required for growth on hibernating bats.

A group of subtilisin serine endopeptidases (MEROPS family S08A) of $P$. destructans have previously been described as PdSP1, PdSP2, ${ }^{25}$ and one of them (PdSP2; destructin-1) was subsequently shown to degrade collagen, suggesting a potential role in colonization of bat skin ${ }^{26}$. Using the MEROPS protease database, 230 predicted proteases were identified from the $P$. destructans genome, which represents a $28 \%$ reduction compared to the average of the nonpathogenic Pseudogymnoascus species (average: 321; range: 308-330) (Fig. 2; Supplementary Data 12). The subtilisin proteases appear to be conserved in the Pseudogymnoascus species analyzed here and therefore is consistent with the observation from Vraný et al. ${ }^{27}$ that collagen-degrading enzymes are present in several nonpathogenic soil microorganisms. While proteases are likely to be involved in colonization of bat skin tissue by $P$. destructans, the previously identified subtilisin proteases (e.g., PdSP2; destructin-1) do not appear to have evolved specifically for this purpose. Moreover, PdSP2 has recently been shown to be more highly expressed in laboratory culture medium than during WNS ${ }^{28,29}$, indicating that it may not play a large role during pathogenesis.

Secreted proteins are generally important for fungi as digestion of nutrients using secreted enzymes occurs outside the fungal cell. Recently, fungal effectors have been described in plant pathogens (some of which can enter animal cells), an example of the intricate competitive arms race between pathogen and host (reviewed in refs. ${ }^{30,31}$ ). The predicted secretome of $P$. destructans is also reduced by more than $50 \%$ in comparison to the nonpathogenic Pseudogymnoascus species (452 vs. average of 970 secreted proteins; range: $848-1033$ ) (Fig. 2). The trend of fewer secreted proteins in fungal pathogens of animals has been previously described ${ }^{32,33}$, with one hypothesis being that losing unnecessary secreted proteins is an evolutionary strategy to evade vertebrate immune systems ${ }^{32}$. Previous reports indicate that fungal secretomes can be lineage specific, consistent with the observation that 56 of the 362 predicted soluble secreted proteins in $P$. destructans $(\sim 15 \%)$ do not have orthologs in the nonpathogenic Pseudogymnoascus species.

To visually depict the functional variation between species, we generated count data of InterProScan Domain and Pfam domains for each genome in our study (Supplementary Data 13-14). The resulting matrices were visualized using a nonmetric multidimensional scaling (NMDS) ordination, which consistently identified $P$. destructans as distinct from the nonpathogenic Pseudogymnoascus species (Supplementary Fig. 2). To identify functional groups and processes that were enriched (positively or negatively), which could help explain the shift in functional domains between $P$. destructans and the nonpathogenic 


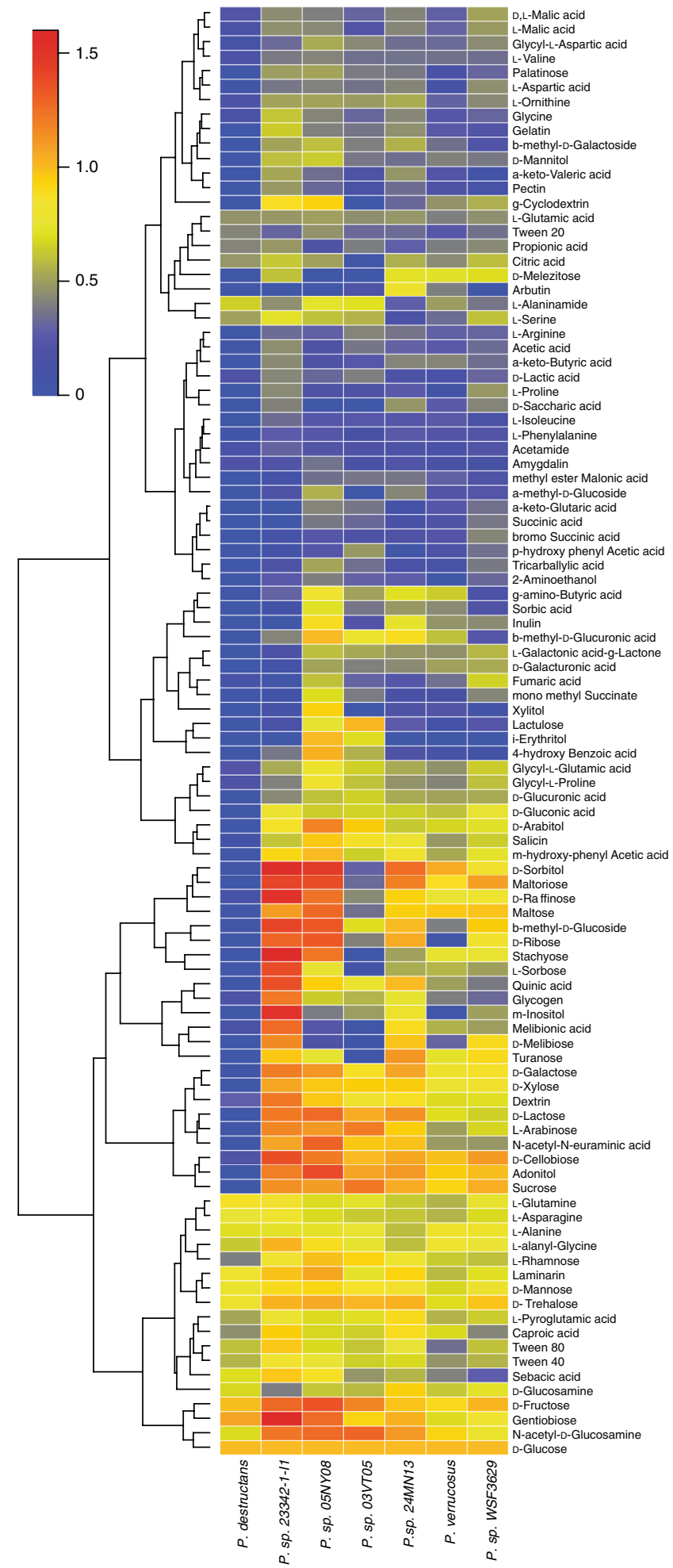

Fig. 3 Heatmap of Biolog phenotypic microarrays testing fungal growth. Carbon utilization from 190 sources was tested to assess the growth of each of seven Pseudogymnoascus species. Growth was quantified using ImageJ ColonyArea plug-in after $15^{\circ} \mathrm{C}$ incubation for 7 days for the nonpathogenic Pseudogymnoascus species and after 14 days for $P$. destructans. Growth for each carbon source is presented as normalized to growth on glucose. These data are derived from a single biological replicate $(n=1)$
Pseudogymnoascus species, we used a Gene Ontology (GO) term enrichment method. As expected and consistent with the ordinations, no enrichment of GO terms was found for any of the six nonpathogenic Pseudogymnoascus species. Two very broad biological processes (BP) were enriched in P. destructans: cellular processes (GO:0009987) and cellular metabolic processes (GO:00044327) (Supplementary Table 3). On the other hand, some more specific BP terms were underrepresented in $P$. destructans: transmembrane transport (GO:0055085), carbohydrate metabolic process (GO:005975), and oxidation-reduction process (GO:0055114). This is consistent with reductions in the secretome, as well as CAZymes using alternate methods as previously discussed. Additionally, the GO enrichment identified a trend in underrepresented BP terms related to transcription (GO:0006351, GO:0097659, and GO:0032774) (Supplementary Table 3). Generally, these data suggest that major genome-level differences between $P$. destructans and the nonpathogenic Pseudogymnoascus species are driven by the large differences in carbohydrate utilization enzymes, as well as a reduction in the putative secretome.

A hallmark of many ascomycete fungi is their ability to produce bioactive small molecules, such as the pharmaceuticals lovastatin and penicillin ${ }^{34}$. These small molecules are also known as secondary metabolites and many are produced by the coordinated effort of gene clusters (genes physically located in proximity to each other on a chromosome). Secondary metabolites have been hypothesized to be involved in niche exploitation by fungi, including evasion of vertebrate immune systems ${ }^{35}$. AntiSMASH v3.0 $0^{36}$ was used to predict secondary metabolite gene clusters from each genome. Based on antiSMASH prediction, $P$. destructans harbors 14 predicted secondary metabolite gene clusters, while the average for the nonpathogenic Pseudogymnoascus species was 27 gene clusters, with a range of 14-36 (Supplementary Table 4, Supplementary Data 15-21). Despite having fewer putative secondary metabolism clusters, the $P$. destructans genome encodes for the two non-ribosomalpolyketide synthetase enzymes required to produce the ironscavenging siderophores that have previously been characterized chemically: ferrichrome and triacetylfusarinine $C^{37}$ (Supplementary Data 15). Additionally, $P$. destructans harbors a putative melanin cluster that has been shown to be involved in pathogenicity in other fungi such as the opportunistic human pathogen Aspergillus fumigatus; four of the six nonpathogenic Pseudogymnoascus species also harbor a similar melanin-like cluster, which in A. fumigatus is physically located in the cell wall of spores of the fungus and has been hypothesized to function as protection from ultraviolet (UV) light and reactive oxygen species (ROS) damage ${ }^{38,39}$. The secondary metabolite arsenal of the Pseudeurotiacae fungi in this study is less than some other groups such as the Eurotiomycetes, which can have 70 or more secondary metabolism clusters. However, several of the secondary metabolites produced by $P$. destructans warrant future study as they could contribute to the development and persistence of WNS in bats.

An interesting characteristic of the $P$. destructans genome is that it contains a large expansion of repetitive DNA sequences, accounting for $38.17 \%$ of the genome, a significant expansion in comparison to the nonpathogenic Pseudogymnoascus species (Table 1). Coinciding with this repeat expansion is a reduction in total gene models, as well as a general trend toward a reduction in several gene families, driven largely by a massive reduction in CAZymes, secreted proteins, and proteases. At the same time, $P$. destructans contains more lineage- specific genes than the nonpathogenic Pseudogymnoascus species (Fig. 2). The observation of expanded repetitive elements and increasing numbers of lineage-specific genes is reminiscent of the lineage- 
specific regions/chromosomes in Fusarium oxysporum ${ }^{40}$. However, in contrast to $F$. oxysporum, analysis of the repetitive sequences across the $P$. destructans genome suggests a uniform distribution (Supplementary Fig. 2A) and lineage-specific genes were not colocalized with repetitive regions (Supplementary Fig. 2B). Similarly to fungal pathogens in the Onygenales, $P$. destructans lacks proteins containing the fungal cellulose-binding domain $(\mathrm{CBM} 18)^{41}$; however, this does not seem to be tied to pathogenicity as 5 of the 6 nonpathogenic Pseudogymnoascus species also lack this functional domain. We were unable to identify protein families that were expanded in $P$. destructans, somewhat reminiscent of Coccidioides, which have very few expanded protein families ${ }^{41}$. This is in stark contrast to the amphibian chytrid pathogens (Batrachochytrium dendrobatidis and Batrachochytrium salamandrivorans), which have undergone an expansion of protease gene families, as well as their genomes en route to pathogenicity ${ }^{42}$. Given the evolutionary distance between the chytrids and ascomycete fungi, it is perhaps not surprising that these devastating fungal pathogens have different evolutionary trajectories.

Light and DNA repair pathways. Taken together, our data suggest that $P$. destructans has been a pathogen of bats for millions of years and thus has likely coevolved in the absence of light. Most organisms that have been found in the absence of light maintain the ability to repair DNA caused by UV light radiation, including most microbes that have been isolated from hibernacula $^{43,44}$. Moreover, a metagenomics analysis of a cave ecosystem identified an overrepresentation of DNA repair enzymes, despite the absence of UV light ${ }^{45}$. We challenged the fungal species studied here with four different DNA-damaging agents that included UV, methyl methanesulfonate (MMS), 4-nitroquinoline (4-NQO), and camptothecin (CPT). While we observed slight differences in sensitivity among species (and even between isolates of $P$. destructans) with 4-NQO and CPT, the most dramatic differential sensitivity was seen with UV light and MMS (Fig. 4a). To further characterize the sensitivity to UV light, we employed a quantitative conidial survival assay and exposed the fungi to three different wavelengths of UV light and three different exposure levels (Fig. 4b). These data show that none of the fungi tested were sensitive to UV-A $(366 \mathrm{~nm})$ light at the dosage tested, while $P$. destructans is differentially sensitive to the higher-energy UV-B $(312 \mathrm{~nm})$ and UV-C (254 nm). Remarkably, a low dose of $5 \mathrm{~mJ} /$ $\mathrm{cm}^{2}$ exposure of UV-C light resulted in only $\sim 15 \%$ survival, while a $10 \mathrm{~mJ} / \mathrm{cm}^{2}$ UV-C exposure resulted in $<1 \%$ survival of $P$. destructans (Fig. 4b).
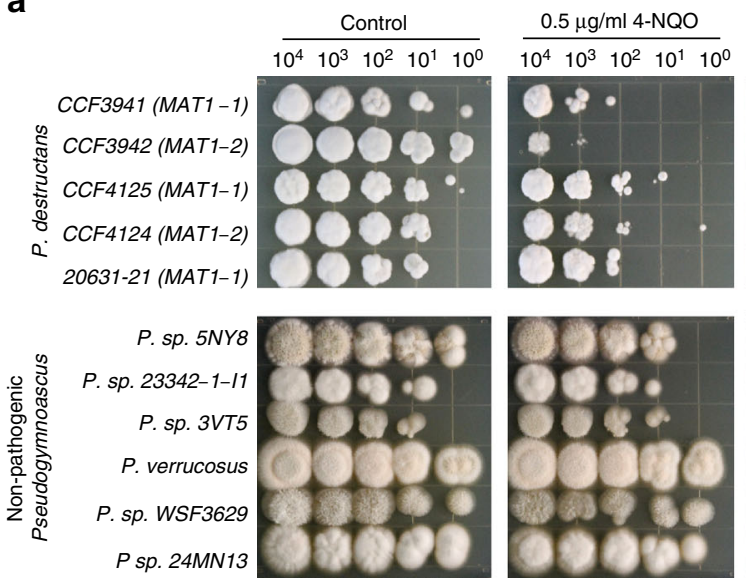
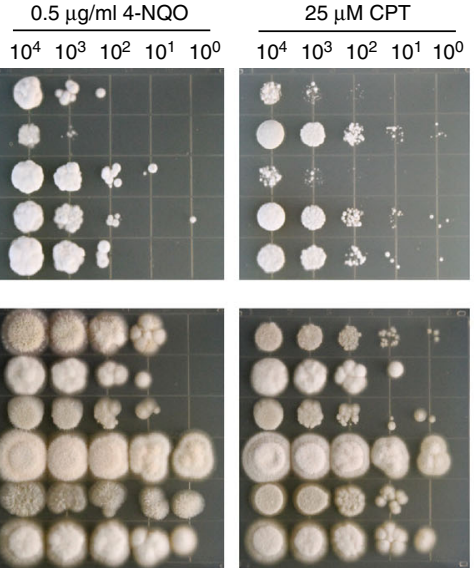
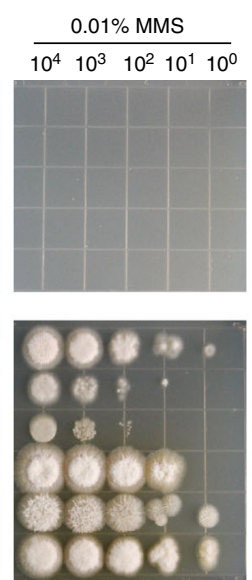
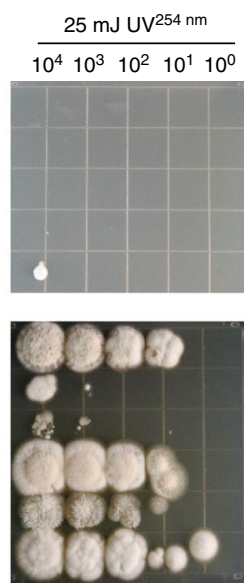

b
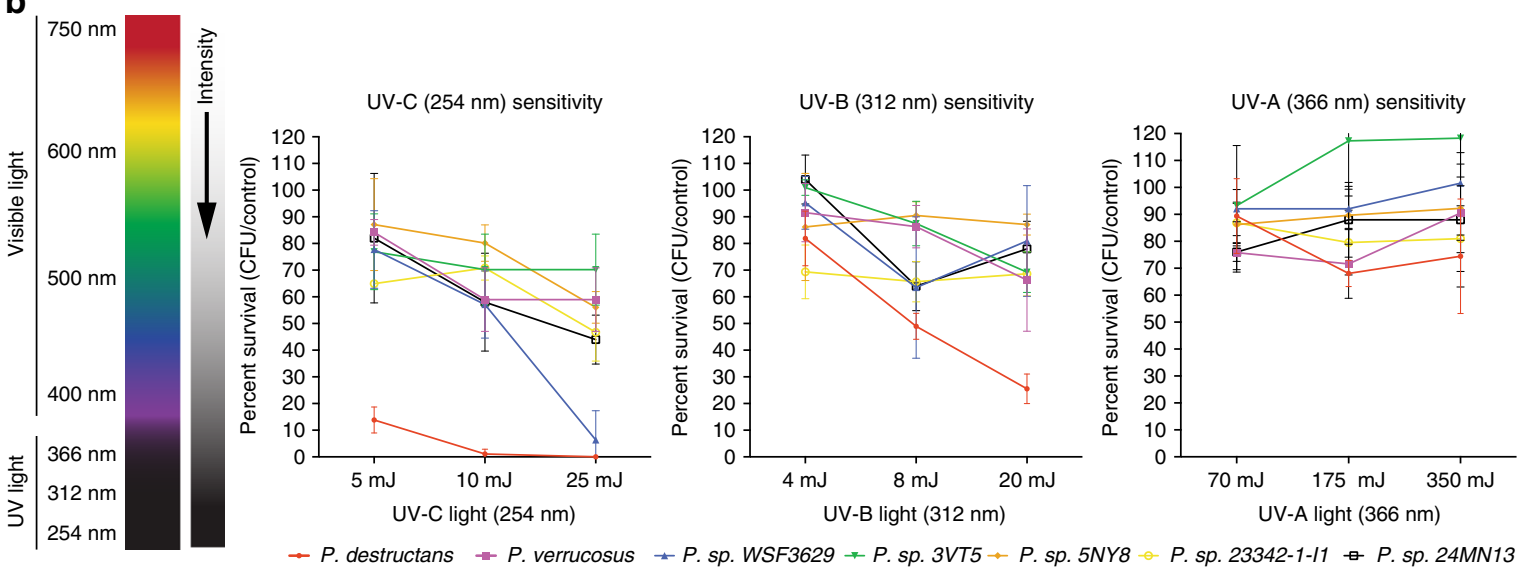

Fig. 4 Sensitivity of Pseudogymnoascus species to DNA-damaging agents. a Qualitative plate assay measuring the effects of four DNA-damaging agents on the growth of each fungal species (4-nitroquinoline (4-NQO), camptothecin (CPT), methyl methanesulfonate (MMS), and 254-nm ultraviolet light (UVC)). Fungal spores were serially diluted, inoculated on appropriate medium, and growth was quantified after 7-day incubation at $15^{\circ} \mathrm{C}$. Multiple isolates of $P$. destructans were tested alongside the nonpathogenic Pseudogymnoascus species. b A quantitative assay using colony-forming units (CFUs) to measure survival of each fungus under different wavelengths of UV light ( $254 \mathrm{~nm}$ (UV-C), $312 \mathrm{~nm}$ (UV-B), and $366 \mathrm{~nm}$ (UV-A)). CFU assays were conducted in biological triplicate $(n=3)$ and error bars represent standard deviations 

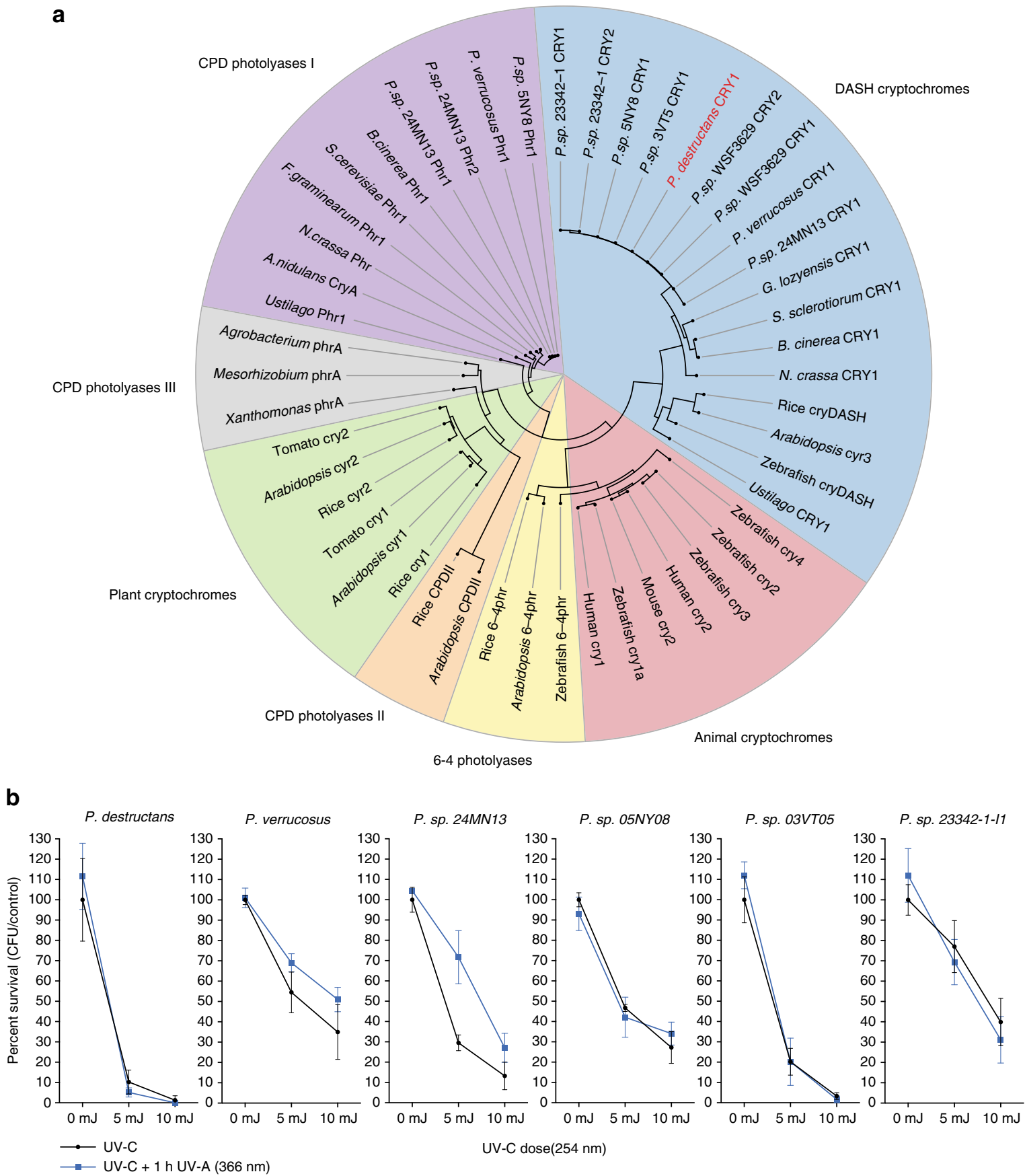

Fig. 5 Blue light-mediated photoreactivation DNA repair is not functional in P. destructans. a Characterization of photolyases in Pseudogymnoascus species based on protein alignment and maximum likelihood phylogeny of well-characterized enzymes. Pseudogymnoascus destructans lacks a canonical CPD photolyase I, although it harbors a cyr-DASH photolyase (VC83_00225). The genomes of Pseudogymnoascus verrucosus, P. sp. 24MN13, and P. sp. 05NY08 contain a CPD photolyase I, whereas all species studied contain at least one cry-DASH ortholog. Proteins identified in ref. ${ }^{66}$ were used for phylogenetic comparison of DNA photolyases. b Photoreactivation experiments comparing survival of germinating conidia exposed to varying doses of UV-C (254 nm) followed by treatment with UV-A $(366 \mathrm{~nm})$ for $1 \mathrm{~h}$ indicate that only $P$. verrucosus and $P$. sp. $24 M N 13$ display increased survival attributed to the activity of CPD photolyase I. No change in survival was detected in $P$. destructans or the other three nonpathogenic Pseudogymnoascus species tested. Interestingly, $P$. sp. 05NY08 did not show an increase in CFU survival despite the fungus harboring a CPD photolyase I enzyme. A similar phenomenon occurs in Aspergillus nidulans, where there was no change in CFU survival in photoreactivation assays in wild-type background, despite the presence of the functional CryA gene (CPD photolyase I) ${ }^{67}$. CFU assays were performed in biological triplicate $(n=3)$ and error bars represent standard deviation

UV light damages DNA by inducing the formation of pyrimidine dimers (cyclobutane dimers and 6-4 photoproducts), while MMS alkylates guanine and adenine nucleotides ${ }^{46}$. Damaged or modified nucleotides result in mispairing and/or replication fork blockage and therefore can result in mutations. Camptothecin's mode of action is similar as it inhibits topoisomerase I function, which results in stalled replication forks; thus, lethal mutations can accumulate during $\mathrm{S}$ phase ${ }^{47}$. On the other hand, 4-NQO induces single base pair mutations with a bias toward guanine to thymine transversions ${ }^{48}$ and thus can cause direct mutagenesis. To combat DNA damage, organisms have employed several DNA repair pathways, including 
photoreactivation, base excision repair (BER), nucleotide excision repair (NER), double-strand break repair, mismatch repair, as well as alternate excision repair (AER) (reviewed in refs. ${ }^{49-51}$ ). Using fission yeast (Schizosaccharomyces pombe) as a model organism, we extracted 169 proteins annotated as involved in DNA repair from www.pombase.org and queried them against the Pseudogymnoascus proteomes using BLAST (Supplementary Data 22). Six putative hits, UVE1, MAG1, MAG2, CENP-X, SWI5, and PIF1, were identified on the basis that they were absent in $P$. destructans but present in most of the six nonpathogenic Pseudogymnoascus. To validate that orthologous proteins were in fact missing as opposed to being misannotated, we queried the corresponding Pfam hidden Markov model (HMM) profiles from (http://www.pfam.org/) using an exhaustive HMM model search in Phyloma (https://github.com/nextgenusfs/ phyloma), which can identify truncated and/or unannotated genes. These results indicated that MAG1, MAG2, and CENP-X orthologs were missing from all Pseudogymnoascus genomes (which is consistent with the low scores of the BLAST hits), while partial matches were found for SWI5 and PIF1, indicating either missed gene models during annotation or nonfunctional proteins. Finally, we were unable to find any trace of UVE1 in the $P$. destructans genome, despite finding clear homologs in the six nonpathogenic Pseudogymnoascus species, suggesting that UVE1 plays a large role in the repair of UV-damaged DNA in Pseudogymnoascus.

$P$. destructans harbors a putative DNA photolyase (VC83_00225); thus, photoreactivation could be a mechanism the fungus uses to repair UV-induced DNA lesions. However, DNA photolyases require light for function and therefore are unlikely to be a major contributor to DNA repair in hibernacula. Preliminary testing of survivability of $P$. destructans incubated in either light or dark indicated that light was insufficient to repair DNA lesions (Supplementary Fig. 3). There are several known classes of DNA photolyases in fungi, including the canonical CPD photolyase I family shown to directly repair cyclobutane dimers, as well as a cyr-DASH family shown to be involved in lightsensing phenotypes ${ }^{52,53}$. A maximum likelihood phylogeny shows that $P$. destructans VC83_00225 is a member of the cry-DASH family of DNA photolyases and is thus unlikely to contribute to direct photorepair of cyclobutane dimers (Fig. 5a). To be thorough, we tested photoreactivation in the laboratory and found no phenotype in $P$. destructans, while two of the nonpathogenic Pseudogymnoascus species that harbored at least one CPD photolyase I ( $P$. verrucosus and P. sp. 24MN13) showed increased survival when exposed to UV-A $(366 \mathrm{~nm})$ light after DNA damage (Fig. 5b).

In fission yeast, UVE1 is a key component of the AER pathway; therefore, our data suggest that most UV-damaged DNA in Pseudogymnoascus is repaired through AER. A small percentage of repair can be attributed to photoreactivation in P. verrucosus and P. sp. 24MN13; however, this appears to not occur in $P$. destructans as it lacks a cyclobutane-dimer photorepair enzyme. The $P$. destructans genome harbors clear homologs for the major enzymes involved in NER pathway (Supplementary Data 22), and thus, more work is needed to determine why the general NER and BER pathways are unable to compensate for loss of AER in $P$. destructans. Nevertheless, the extreme sensitivity to UV-C light in $P$. destructans represents a genetic pathway that could be exploited for active management of WNS of bats. Currently, UV-A $(366 \mathrm{~nm})$ light is being used as a WNS noninvasive field diagnostic tool because the characteristic cupping skin lesions fluoresce under this wavelength ${ }^{54}$. While UV-A light tested under laboratory conditions had no effect on survival of $P$. destructans (Fig. 4), the use of UV-A as a diagnostic tool suggests that treating individual bats with a dosage of UV-C light is feasible. The relatively low dose $\left(\sim 10 \mathrm{~mJ} / \mathrm{cm}^{2}\right)$ of UV-C light required to kill $P$. destructans conidia could be applied to bats in a few seconds of exposure from a portable light source. More work is needed to understand the physiological effect of UV-C light on bats; however, it is encouraging that treatment of mammal fungal skin/ nail infections with UV-C light has been used for dermatophyte fungi causing onychomycosis ${ }^{55}$, as well as wound infections caused by Candida albicans ${ }^{56}$.

\section{Discussion}

WNS represents one of the most severe wildlife diseases ever recorded. Currently, there are no practical treatment options for containing the spread of WNS in North America and thus the fungus has moved rapidly across the continent. Survivor populations of affected bat species can still be found near the disease outbreak epicenter, so that there is hope that species extinction will be avoided, albeit with a prolonged recovery ${ }^{57}$. However, the long-term effects on ecosystems involving bats will not be understood for decades. Comparative genomics analyses presented here suggest that $P$. destructans is likely a true fungal pathogen of bats, evolving alongside Eurasian bat species for millions of years. The annotated genome of $P$. destructans in addition to several nonpathogenic closely related species provides a framework for understanding the pathobiology of WNS. The serendipitous discovery that $P$. destructans lacks a UVE1 homolog and is therefore extremely sensitive to pyrimidine dimer inducing DNA-damaging agents is a vulnerability that could be exploited for WNS management.

\section{Methods}

Growth conditions, DNA extraction, and sequencing. Fungal strains were grown in liquid-stationary culture ${ }^{58}$ for several days at $15^{\circ} \mathrm{C}$, mycelia were lyophilized, and subsequently genomic DNA (gDNA) was extracted as previously described ${ }^{59}$ High-molecular-weight gDNA was confirmed by gel electrophoresis. For sequencing on the Ion Torrent Personal Genome Machine (PGM), a random 400-bp size fractionated library was constructed using Ion Plus Fragment Library Kit (\#4471257), templated using the Ion PGM Template OT2 400 Kit (\#4479878), loaded on a $318 \mathrm{v} 2$ chip (\#4484354, and sequenced using the Ion 400 bp Sequencing Kit (\#4482002) (all kits were used according to the manufacturer's recommendations). Library construction for sequencing using the Illumina GAx II and Illumina MiSeq was done using the NEBNext Library Prep Kit, purified using QIAQuick Cartridge Kit, and size fractionated using E-gel 2\% size select gel electrophoresis system (ThermoFisher). For obtaining RNA-seq reads to assist with genome annotation, fungi were grown in liquid-shaking cultures at $15^{\circ} \mathrm{C}$ for 3 days, mycelia were lyophilized, total RNA was extracted using TriZol (Invitrogen), and polyadenylated RNA was selected using the DynaBeads PolyA Cleanup Kit (Ambion). The poly-A RNA was then used to make libraries using the Ion RNA-seq Kit 2.0 and sequenced on the Ion Torrent PGM using Ion 200 bp Sequencing Kit (\#4482006).

Genome assembly. Initial attempts to build a high-quality assembly for $P$. destructans using paired-end MiSeq $(2 \times 250 \mathrm{bp})$ data resulted in heavily fragmented assemblies with several different assembly software (Discovar DeNovo, Abyss, Spades, CLC, etc.). We generated a highly contiguous assembly using a combination of PacBio reads, MiSeq, Roche 454 mate-pair reads, and Sanger end sequences from a $100-\mathrm{kb}$ BAC library ${ }^{14}$. For five of the nonpathogenic Pseudogymnoascus species (UAMH10579, 03VT05, 05NY08, WSF3629, and 23342-1-I1), acceptable assemblies were generated using the de novo assembler in CLC Genomics Workbench 7.5 (Qiagen) using paired-end MiSeq reads $(2 \times 300 \mathrm{bp})$. For Pseudogymnoascus sp. $24 \mathrm{MN} 13$, the paired-end Illumina $(2 \times 100 \mathrm{bp}$; GAIIx $)$ data were found to have some bacterial contamination, thus, we generated a second sequencing library from a clean gDNA extraction for the Ion Torrent PGM, which was used for an initial assembly and scaffolding using the paired-end Illumina reads. Contamination was removed from the assemblies using DeconSeq ${ }^{60}$ and was subsequently error corrected using Pilon ${ }^{61}$. Small repetitive contigs from each assembly were identified using Mummer ${ }^{62}$ and were removed if they were $95 \%$ identical over $95 \%$ of their length with other contigs in the assembly (achieved using "funannotate clean" command).

Genome annotation and functional characterization. Detailed information on annotation and functional characterization is presented in Supplementary Methods. 
Biolog phenotyptic microarray. Pseudogymnoascus species were tested for the ability to utilize 190 different carbon sources in the Biolog Phenotyptic Microarray platform (plates PM1 and PM2A). Fungal spores at a density of $1 \times 10^{4}$ spores were inoculated into $100 \mu \mathrm{l}$ of carbon-free minimal medium (MM lacking glucose ${ }^{63}$ ) and then subsequently aliquoted into 96-well Biolog plates (PM1 and PM2A). Cultures were grown at $15^{\circ} \mathrm{C}$ and the nonpathogenic Pseudogymnoascus species were incubated for 7 days, while $P$. destructans was incubated for 14 days. The plates were then photographed and growth was quantified using the Fiji/ImageJ plug-in ColonyArea $1.5^{64}$. Relative growth was then normalized to growth on glucose and plotted in heatmap. 2 from the gplots package in $\mathrm{R}^{65}$.

DNA damage assays. Conidia were harvested from fungal isolates from solid medium agar plates using sterile $0.01 \%$ Tween- 80 water, purified by filtering over sterile miracloth (EMD Millipore \#475855), and enumerated using a hemocytometer. For spot plate assays, conidia were serially diluted and $5 \mu \mathrm{l}$ was pipetted onto the surface of glucose minimal agar medium ${ }^{63}$. Treatment with UV light was accomplished by exposing the spotted agar plate to $25 \mathrm{~mJ} / \mathrm{cm}^{2}$ of UV-C $(254 \mathrm{~nm})$ light using a UV cross-linker (UVP CL-1000). Sensitivity to chemical DNA mutagens was done by preparing GMM agar medium that contained $25 \mu \mathrm{M} \mathrm{CPT}$, $0.01 \% \mathrm{MMS}$, or $0.5 \mu \mathrm{g} / \mathrm{ml}$ of 4 -nitroquinolone (4-NQO). Control and treated plates were incubated at $15^{\circ} \mathrm{C}$ for 1 week prior to imaging. Colony-forming unit (CFU) assays were done by spread plating $\sim 50$ conidia on the surface of a GMM agar plate and were subsequently exposed to UV light in a UV cross-linker, and surviving colonies were counted after incubation at $15{ }^{\circ} \mathrm{C}$ for 1 week. Quantification of photoreactivation for each species was done using a very similar CFU assay comparing varying UV-C treatments followed by 1-h exposure to UV-A $(366 \mathrm{~nm})$ light in a UV cross-linker. Conidia were allowed to germinate for $24 \mathrm{~h}$ in the dark prior to UV-C $(254 \mathrm{~nm})$ treatments and subsequent UV-A $(366 \mathrm{~nm})$ treatment. Surviving colonies were counted after incubation in the dark at an appropriate temperature $\left(15^{\circ} \mathrm{C}\right.$ for $P$. destructans and $25^{\circ} \mathrm{C}$ for nonpathogenic Pseudogymnoascus species). All CFU assays were done in biological triplicates $(n=3)$ for each experimental condition.

Data availability. Sequencing data and genome assemblies are available via NCBI under BioProject PRJNA276926 and SRA project SRP055906. All other relevant data supporting the findings of the study are available in this article and its Supplementary Information files, or from the corresponding author upon request.

Received: 29 March 2017 Accepted: 30 November 2017 Published online: 02 January 2018

\section{References}

1. Lorch, J. M. et al. First detection of bat white-nose syndrome in western North America. mSphere 1, e00148-16 (2016).

2. Frick, W. F. et al. An emerging disease causes regional population collapse of a common North American bat species. Science 329, 679-682 (2010).

3. Gargas, A., Trest, M. T., Christensen, M., Volk, T. J. \& Blehert, D. S. Geomyces destructans sp. nov. associated with bat white-nose syndrome. Mycotaxon 108, 147-154 (2009).

4. Minnis, A. M. \& Lindner, D. L. Phylogenetic evaluation of Geomyces and allies reveals no close relatives of Pseudogymnoascus destructans, comb. nov., in bat hibernacula of eastern North America. Fungal Biol. 117, 638-649 (2013).

5. Verant, M. L., Boyles, J. G., Waldrep, W., Wibbelt, G. \& Blehert, D. S. Temperature-dependent growth of Geomyces destructans, the fungus that causes bat white-nose syndrome. PLoS ONE 7, e46280 (2012).

6. Cryan, P. M., Meteyer, C. U., Boyles, J. G. \& Blehert, D. S. Wing pathology of white-nose syndrome in bats suggests life-threatening disruption of physiology. BMC Biol. 8, 135-135 (2010).

7. Reeder, D. M. et al. Frequent arousal from hibernation linked to severity of infection and mortality in bats with white-nose syndrome. PLoS ONE 7, e38920 (2012).

8. Lorch, J. M. et al. Experimental infection of bats with Geomyces destructans causes white-nose syndrome. Nature 480, 376-378 (2011).

9. Verant, M. L. et al. White-nose syndrome initiates a cascade of physiologic disturbances in the hibernating bat host. BMC Physiol. 14, 10 (2014).

10. Warnecke, L. et al. Inoculation of bats with European Geomyces destructans supports the novel pathogen hypothesis for the origin of white-nose syndrome. Proc. Natl. Acad. Sci. USA 109, 6999-7003 (2012).

11. Puechmaille, S. J. et al. White-nose syndrome: is this emerging disease a threat to European bats? Trends Ecol. Evol. 26, 570-576 (2011).

12. Lorch, J. M. et al. A culture-based survey of fungi in soil from bat hibernacula in the eastern United States and its implications for detection of Geomyces destructans, the causal agent of bat white-nose syndrome. Mycologia https://doi. org/10.3852/12-207 (2012).
13. Lorch, J. M. et al. Distribution and environmental persistence of the causative agent of white-nose syndrome, Geomyces destructans, in bat hibernacula of the eastern United States. Appl. Environ. Microbiol. 79, 1293-1301 (2013).

14. Drees, K. P. et al. Use of multiple sequencing technologies to produce a highquality genome of the fungus Pseudogymnoascus destructans, the causative agent of bat white-nose syndrome. Genome Announc. 4, e00445-16 (2016).

15. Simão, F. A., Waterhouse, R. M., Ioannidis, P., Kriventseva, E. V. \& Zdobnov, E. M. BUSCO: assessing genome assembly and annotation completeness with single-copy orthologs. Bioinformatics 31, 3210-3212 (2015).

16. Beimforde, C. et al. Estimating the phanerozoic history of the Ascomycota lineages: combining fossil and molecular data. Mol. Phylogen. Evol. 78, 386-398 (2014).

17. Sanderson, M. J. r8s: inferring absolute rates of molecular evolution and divergence times in the absence of a molecular clock. Bioinformatics 19, 301-302 (2003).

18. Novacek, M. J. Evidence for echolocation in the oldest known bats. Nature 315, 140-141 (1985).

19. Springer, M. S., Teeling, E. C., Madsen, O., Stanhope, M. J. \& de Jong, W. W. Integrated fossil and molecular data reconstruct bat echolocation. Proc. Natl. Acad. Sci. USA 98, 6241-6246 (2001).

20. Ruedi, M. \& Mayer, F. Molecular systematics of bats of the genus Myotis (Vespertilionidae) suggests deterministic ecomorphological convergences. Mol Phylogenet. Evol. 21, 436-448 (2001).

21. Lechner, M. et al. Orthology detection combining clustering and synteny for very large datasets. PLoS ONE 9, e105015 (2014).

22. Yang, Z. PAML 4: phylogenetic analysis by maximum likelihood. Mol. Biol. Evol. 24, 1586-1591 (2007)

23. Zhao, Z., Liu, H., Wang, C. \& Xu, J.-R. Correction: comparative analysis of fungal genomes reveals different plant cell wall degrading capacity in fungi. BMC Genom. 15, 6 (2014).

24. Wilson, M. B., Held, B. W., Freiborg, A. H., Blanchette, R. A. \& Salomon, C. E. Resource capture and competitive ability of non-pathogenic Pseudogymnoascus spp. and $P$. destructans, the cause of white-nose syndrome in bats. PLoS ONE 12, e0178968 (2017).

25. Pannkuk, E. L., Risch, T. S. \& Savary, B. J. Isolation and identification of an extracellular subtilisin-like serine protease secreted by the bat pathogen Pseudogymnoascus destructans. PLoS ONE 10, e0120508 (2015).

26. O'Donoghue, A. J. et al. Destructin-1 is a collagen-degrading endopeptidase secreted by Pseudogymnoascus destructans, the causative agent of white-nose syndrome. Proc. Natl. Acad. Sci. USA 112, 7478-7483 (2015).

27. Vraný, B., Hnátková, Z. \& Lettl, A. Occurrence of collagen-degrading microorganisms in associations of mesophilic heterotrophic bacteria from various soils. Folia Microbiol. 33, 458-461 (1988).

28. Reeder, S. M. et al. Pseudogymnoascus destructans transcriptome changes during white-nose syndrome infections. Virulence $\mathbf{0}$, https://doi.org/10.1080/ 21505594.2017.1342910 (2017).

29. Field, K. A. et al The white-nose syndrome transcriptome: activation of antifungal host responses in wing tissue of hibernatinglittle brown Myotis. PLoS Pathog. 11, e1005168 (2015).

30. Petre, B. \& Kamoun, S. How do filamentous pathogens deliver effector proteins into plant cells? PLoS Biol. 12, e1001801 (2014).

31. Kale, S. D. \& Tyler, B. M. Entry of oomycete and fungal effectors into plant and animal host cells. Cell. Microbiol. 13, 1839-1848 (2011)

32. Krijger, J.-J., Thon, M. R., Deising, H. B. \& Wirsel, S. G. R. Compositions of fungal secretomes indicate a greater impact of phylogenetic history than lifestyle adaptation. BMC Genom. 15, 722 (2014).

33. Kim, K.-T. et al. Kingdom-wide analysis of fungal small secreted proteins (SSPs) reveals their potential role in host association. Front. Plant Sci. 7, 186 (2016).

34. Keller, N. P., Turner, G. \& Bennett, J. W. Fungal secondary metabolism - from biochemistry to genomics. Nat. Rev. Microbiol. 3, 937-947 (2005).

35. Scharf, D. H., Heinekamp, T. \& Brakhage, A. A. Human and plant fungal pathogens: the role of secondary metabolites. PLoS Pathog. 10, e1003859 (2014).

36. Weber, T. et al. antiSMASH 3.0-a comprehensive resource for the genome mining of biosynthetic gene clusters. Nucleic Acids Res. 43, W237-W243 (2015).

37. Mascuch, S. J. et al. Direct detection of fungal siderophores on bats with whitenose syndrome via fluorescence microscopy-guided ambient ionization mass spectrometry. PLoS ONE 10, e0119668 (2015).

38. Jahn, B. et al. Isolation and characterization of a pigmentless-conidium mutant of Aspergillus fumigatus with altered conidial surface and reduced virulence. Infect. Immun. 65, 5110-5117 (1997).

39. Langfelder, K., Streibel, M., Jahn, B., Haase, G. \& Brakhage, A. A. Biosynthesis of fungal melanins and their importance for human pathogenic fungi. Fungal Genet. Biol. 38, 143-158 (2003).

40. Ma, L.-J. et al. Comparative genomics reveals mobile pathogenicity chromosomes in Fusarium. Nature 464, 367-373 (2010). 
41. Sharpton, T. J. et al. Comparative genomic analyses of the human fungal pathogens Coccidioides and their relatives. Genome Res. 19, 1722-1731 (2009).

42. Farrer, R. A. et al. Genomic innovations linked to infection strategies across emerging pathogenic chytrid fungi. Nat. Commun. 8, 14742 (2017).

43. Arrage, A. A., Phelps, T. J., Benoit, R. E. \& White, D. C. Survival of subsurface microorganisms exposed to UV radiation and hydrogen peroxide. Appl. Environ. Microbiol. 59, 3545-3550 (1993).

44. Snider, J., Goin, C., Miller, R., Boston, P. \& Northup, D. Ultraviolet radiation sensitivity in cave bacteria: evidence of adaptation to the subsurface? Int. J. Speleol. 38, 11-22 (2009).

45. Ortiz, M. et al. Making a living while starving in the dark: metagenomic insights into the energy dynamics of a carbonate cave. ISME J. 8, 478-491 (2014).

46. Beranek, D. T. Distribution of methyl and ethyl adducts following alkylation with monofunctional alkylating agents. Mutat. Res. 231, 11-30 (1990).

47. Fiorani, P. \& Bjornsti, M. A. Mechanisms of DNA topoisomerase I-induced cell killing in the yeast Saccharomyces cerevisiae. Ann. N. Y. Acad. Sci. 922, 65-75 (2000).

48. Downes, D. J. et al. Characterization of the mutagenic spectrum of 4-nitroquinoline 1-oxide (4-NQO) in Aspergillus nidulans by whole genome sequencing. G3 4, 2483-2492 (2014).

49. Yasui, A. Alternative excision repair pathways. Cold Spring Harb. Perspect. Biol. 5, a012617 (2013)

50. Dexheimer, T. S. DNA Repair of Cancer Stem Cells 19-32 (Springer, Netherlands, 2012).

51. Marteijn, J. A., Lans, H., Vermeulen, W. \& Hoeijmakers, J. H. J. Understanding nucleotide excision repair and its roles in cancer and ageing. Nat. Rev. Mol. Cell Biol. 15, 465-481 (2014).

52. Cohrs, K. C. \& Schumacher, J. The two cryptochrome/photolyase family proteins fulfill distinct roles in DNA photorepair and regulation of conidiation in the gray mold fungus Botrytis cinerea. Appl. Environ. Microbiol. 83, AEM.00812-17 (2017).

53. Froehlich, A. C. et al. Genetic and molecular characterization of a cryptochrome from the filamentous fungus Neurospora crassa. Eukaryot. Cell 9 , 738-750 (2010).

54. Turner, G. G. et al. Nonlethal screening of bat-wing skin with the use of ultraviolet fluorescence to detect lesions indicative of white-nose syndrome. $J$. Wildl. Dis. 50, 566-573 (2014).

55. Dai, T., Tegos, G. P., Rolz-Cruz, G., Cumbie, W. E. \& Hamblin, M. R. Ultraviolet $C$ inactivation of dermatophytes: implications for treatment of onychomycosis. Br. J. Dermatol. 158, 1239-1246 (2008).

56. Dai, T. et al. Ultraviolet-C light for treatment of Candida albicans burn infection in mice. Photochem. Photobiol. 87, 342-349 (2011).

57. Langwig, K. E. et al. Resistance in persisting bat populations after white-nose syndrome invasion. Philos. Trans. R. Soc. Lond. B Biol. Sci. 372, 20160044 (2017).

58. Champe, S. P. \& el-Zayat, A. A. Isolation of a sexual sporulation hormone from Aspergillus nidulans. J. Bacteriol. 171, 3982-3988 (1989).

59. Palmer, J. M. et al. Molecular characterization of a heterothallic mating system in Pseudogymnoascus destructans, the fungus causing white-nose syndrome of bats. G3 4, 1755-1763 (2014).

60. Schmieder, R. \& Edwards, R. Fast identification and removal of sequence contamination from genomic and metagenomic datasets. PLoS ONE 6, e17288 (2011).

61. Walker, B. J. et al. Pilon: an integrated tool for comprehensive microbial variant detection and genome assembly improvement. PLOS ONE 9, e112963 (2014).

62. Kurtz, S. et al. Versatile and open software for comparing large genomes. Genome Biol. 5, R12 (2004).
63. Shimizu, K. \& Keller, N. P. Genetic involvement of a cAMP-dependent protein kinase in a $\mathrm{G}$ protein signaling pathway regulating morphological and chemical transitions in Aspergillus nidulans. Genetics 157, 591-600 (2001).

64. Guzmán, C., Bagga, M., Kaur, A., Westermarck, J. \& Abankwa, D. ColonyArea: an ImageJ plugin to automatically quantify colony formation in clonogenic assays. PLoS ONE 9, e92444 (2014).

65. R Core Team. R: A Language and Environment for Statistical Computing https://www.r-project.org/ (Vienna, Austria, 2016).

66. Tagua, V. G. et al. Fungal cryptochrome with DNA repair activity reveals an early stage in cryptochrome evolution. Proc. Natl. Acad. Sci. USA 112, 15130-15135 (2015).

67. Bayram, O., Biesemann, C., Krappmann, S., Galland, P. \& Braus, G. H. More than a repair enzyme: Aspergillus nidulans photolyase-like CryA is a regulator of sexual development. Mol. Biol. Cell 19, 3254-3262 (2008).

\section{Acknowledgements}

Funding was provided to J.M.P. and D.L.L. by the US Fish \& Wildlife grant F14PG00132 and to K.P.D. and J.T.F. by US Fish \& Wildlife grant F14AP00644. Additional funding and support for J.M.P. and D.L.L. were provided by the US Forest Service, Northern Research Station.

\section{Author contributions}

J.M.P., K.P.D., J.T.F., and D.L.L. conceived the study; K.P.D. and J.T.F. performed wholegenome sequencing, K.P.D. conducted hybrid genome assembly of $P$. destructans, JMP conducted genome assembly, annotation, genome-level comparisons, and conducted laboratory experiments; and J.M.P. wrote the paper with input from K.P.D., J.T.F., and D. L.L.

\section{Additional information}

Supplementary Information accompanies this paper at https://doi.org/10.1038/s41467017-02441-z.

Competing interests: The authors declare no competing financial interests.

Reprints and permission information is available online at http://npg.nature.com/ reprintsandpermissions/

Publisher's note: Springer Nature remains neutral with regard to jurisdictional claims in published maps and institutional affiliations.

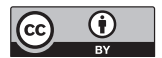

Open Access This article is licensed under a Creative Commons Attribution 4.0 International License, which permits use, sharing, adaptation, distribution and reproduction in any medium or format, as long as you give appropriate credit to the original author(s) and the source, provide a link to the Creative Commons license, and indicate if changes were made. The images or other third party material in this article are included in the article's Creative Commons license, unless indicated otherwise in a credit line to the material. If material is not included in the article's Creative Commons license and your intended use is not permitted by statutory regulation or exceeds the permitted use, you will need to obtain permission directly from the copyright holder. To view a copy of this license, visit http://creativecommons.org/ licenses/by/4.0/.

(C) The Author(s) 2017 